\title{
Simple method to determine the efficiency of a cream used for skin protection against solvents
}

\author{
M. GUILLEMIN, J. C. MURSET, M. LOB, and J. RIQUEZ \\ Department of Occupational Health and Industrial Hygiene, Institute of Social and Preventive \\ Medicine, University, Lausanne, Switzerland
}

\begin{abstract}
Guillemin, M., Murset, J. C., Lob, M., and Riquez, J. (1974). British Journal of Industrial Medicine, 31, 310-316. Simple method to determine the efficiency of a cream used for skin protection against solvents. Most of the industrial organic solvents coming into contact with the skin are absorbed through it and enter the blood. They can then be detected in the breath, the analysis of which makes it easy to evaluate skin absorption. Such an evaluation can be made with and without the use of a skin protective (barrier) cream. A comparison of the results allows the efficiency of the cream to be tested.

An example of the application of this method is described. The solvent was toluene and the cream tested was composed mainly of talc, glycerol, and water. Ten male volunteers exposed their hands to liquid toluene for 10 minutes. The elimination of the solvent was followed by breath analysis up to 3 hours after the exposure. Two or more sets of exposure were made under different conditions with and without the protective cream.

The results show a good standard of efficiency when the cream is carefully and deeply applied. The efficiency of the cream was determined by testing the difference between the decay curves obtained with and without the barrier cream. A relative efficiency was estimated as a percent diminution of solvent excretion by taking the excretion after exposure of unprotected skin as $100 \%$.

It is shown that skin absorption for toluene compared with lung absorption may be important and has to be estimated where total exposure is concerned. The method devised here appears to offer simplicity. It is presented as a laboratory test to show the intrinsic value of the cream but its applicability in the field is also considered.
\end{abstract}

Protective creams, also named barrier creams or invisible gloves, are widely used wherever handling irritant or hazardous compounds is impossible with gloves. Sometimes gloves do not resist the action of a compound or they reduce dexterity or touch too much. Protective creams are employed in a very large number of fields, for instance, photography, electronics, plastics, printing industries, and so on.

${ }^{1}$ Office de Statistique de l'Etat de Vaud, Lausanne, Switzerland
Among substances that damage the skin, or that are absorbed through it, solvents take the first place (Schmid, 1969). The hazard they present is twofold: (1) local dermal lesions and (2) toxic action on other organs after percutaneous absorption.

The methods actually in use to evaluate the extent to which a cream acts as a real barrier against solvents are based on rather complicated and specialized dermatological techniques (Spruit, Malten, Lipmann, and The Poo Liang, 1970; Wahlberg, 1971). In practice on the other hand, it is very difficult for an occupational physician or for an 
industrial hygienist to decide whether a cream is appropriate or not. The development of an easy test appeared therefore to be very useful.

Contact between skin and solvent creates physical and chemical reactions which can result in the penetration of the solvent through the skin. Absorption takes place through both the hair follicles and the intervening skin (Cooke, 1966). Some degree of lipid solubility is necessary for the solvent to penetrate the cell membrane, and the lipid-water distribution coefficient is in general an indication of the capacity for diffusion through the cell (Cooke, 1966). Consequently the solvent can enter the blood and be carried in the circulatory system. In the lungs the equilibrium existing between the concentration of the solvent in the blood and its concentration in alveolar air, according to Henry's law, makes it possible to detect the solvent in the breath. This is an easy way to evaluate absorption through the skin (Stewart and Dodd, 1964).

Dermatosis or irritation of the skin are closely related to percutaneous absorption since both may occur when contact between skin and solvent is established. The method developed in this study consists in comparing the absorption of a solvent when the skin of the hands is protected with a barrier cream and when it is not. The cream tested and the solvent were chosen because of their large-scale use in printing shops.

Surveys have shown that ventilation in these shops was in general well designed and that exposure to toluene was chiefly due to skin contact. Moreover, in the printing industry the workmen very frequently use the same solvent that is employed for other operations (cleaning the machinery, diluting the inks, etc.) to remove inks and dyes from their hands. It was therefore very important to know if the protective cream used here acted as an efficient barrier.

\section{Materials and methods}

\section{Skin exposure}

Ten male volunteers (laboratory staff) 21 to 56 years of age, whose hands were free from cuts or any skin abnormalities, exposed their hands to pure liquid toluene for 10 minutes. The solvent was purchased from Merck (Darmstadt, Germany) and its purity was controlled by gas chromatography (G.C.). A simple geometrical estimation was made of the area of skin exposed. Both hands were immersed up to the wrist in large vials whose tops were closed by a sheet of rubber ( $1 \mathrm{~mm}$ thick) with two holes adjusted to the volunteers' forearms. This was to prevent toluene vapours from escaping from the vials. To avoid any lung exposure, masks with activated charcoal filters (model Comfo, MSA USA) were worn during the whole exposure.

At the end of the exposure the hands were washed thoroughly with soap and water and treated with an appropriate paste to soothe the skin.
The barrier cream (Lorilleux No. 1906, Berne, Switzerland) was composed mainly of glycerol, talc, and water. Two ways of applying the barrier cream were tested:

(1) deep application, the cream being thoroughly massaged into the hands;

(2) superficial application, without lengthy massage, but with care taken to distribute the cream evenly over the skin.

Every volunteer exposed his hands at least twice with an interval of two weeks between the experiments. The first exposure was carried out without the barrier cream and the second one with the cream. Three subjects repeated the experiments to check the reproducibility of the results, i.e., to see if the same exposures give the same elimination curves.

\section{Breath analysis}

Immediately after the skin exposure and handwashing, breath analyses were carried out six to 10 times during a period of two to three hours to obtain the curve showing the elimination of toluene. For practical considerations the interval between the analyses was not constant. This was not critical since the results were well reproducible (low dispersion).

In a few cases breath analysis was carried out before exposure, to be sure that no unexpected pre-exposure had occurred.

Toluene in breath was analysed by G.C. with a Perkin Elmer instrument PE-900. The analysis column consisted of $2 \mathrm{~m}$ long glass tubing (internal diameter $\frac{1}{8}$ inch (3.2 $\mathrm{mm})$ ) filled with chromosorb G (AW-DMCS) $(80 / 100$ mesh) coated with $4 \%$ Tween 80 . The manifold was heated to $160^{\circ} \mathrm{C}$. The detection was made by flame ionization with an air $\left(80 \% \mathrm{~N}_{2}+20 \% \mathrm{O}_{2}\right)$ flow of $500 \mathrm{ml} /$ min and an hydrogen flow of $65 \mathrm{ml} / \mathrm{min}$. Nitrogen was used as the carrier gas at $15 \mathrm{ml} / \mathrm{min}$. The injection was made with a gas valve equipped with a calibrated $1 \mathrm{ml}$ sample loop heated to $150^{\circ} \mathrm{C}$. The retention time of toluene was 2 minutes and the sensitivity $0.01 \mathrm{ppm}$.

A calibration curve for toluene in the range of 0.1 to $10 \mathrm{ppm}$ was obtained by preparing known concentrations of the solvent in air in a spherical glass flask having a volume of 53.61 . This device was equipped with an injection part for introducing accurately weighed liquid solvent, a heater covered with glass, a thermometer, a glass stirrer, and an expansion balloon (Tedlar) to expel the air through an exit connected to the G.C., without changing the concentration inside the flask. For small concentrations it was not possible to weigh pure liquid toluene accurately. For that, a known solution of toluene in dichloromethane was used. This chlorinated solvent did not interfere with the G.C. analysis."

Samples of alveolar air were obtained by means of a mouthpiece equipped with a glass device with two valves, one for inhaling and the other for exhaling. One part of the valve on the expiration side was directly connected to the G.C. instrument described above. A few volunteers had some difficulty in learning the breathing technique but a wrong sampling was obviously shown by too low a value in the toluene concentration.

No correction was applied to the results to obtain the real toluene concentration in alveoli at $37^{\circ} \mathrm{C}$ and $100 \%$ relative humidity. 
Statistical methods

In order to obtain a simple mathematical form for the elimination curves a regression analysis was applied after appropriate transformation of variables. Three equations were tried:

$$
\begin{aligned}
& \mathbf{Y}=\mathbf{A}+\mathbf{B} / \mathbf{x} \\
& \mathbf{Y}=\mathbf{A}+\mathbf{B} / \mathbf{x}^{\mathbf{2}} \\
& \mathbf{Y}=\mathbf{A} \mathbf{e}^{\mathbf{B x}}
\end{aligned}
$$

$\mathrm{Y}=$ toluene concentration (ppm)

$\mathrm{X}=$ time after exposure $(\mathrm{min})$.

To decide which form was the best approximation, each individual experimental curve was tested by its correlation coefficient $r$ (hypothesis $r=0$ ) (Geigy, 1972) and the total results for each equation were tested for homogeneity of $\mathbf{r}\left(\chi^{2}\right.$ test). It was not surprising to see that the exponential form ( $\left.\mathrm{Y}=\mathrm{Ae}^{\mathrm{Bx}}\right)$ was the most suitable.

The best fit of this form was obtained for $x$ values between 20 and 120 minutes. The $A$ constant is the toluene concentration at time zero and $B$ the coefficient of the excretion rate (excretion constant) which gives also the slope of the straight line $\ln \mathrm{Y}=\ln \mathrm{A}+\mathrm{Bx}$.

For each experiment there are two lines, the first $\ln Y_{I}=\ln A_{I}+B_{I} \times_{I}$ for skin exposure with bare hands, and the second $\ln Y_{I I}=\ln A_{I I}+B_{I I} \times$ II for skin exposure with protected hands. For the overall results these regression lines are parallel $\left(\mathrm{B}_{\mathrm{I}}=\mathrm{B}_{\mathrm{II}}\right)$ but a difference may exist for individual experiments due to the greater dispersion of the values in the second experiment (see discussion below). A mean $\bar{B}$ can be estimated for each volunteer by the formula:

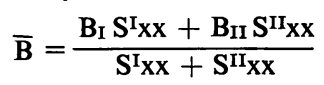

where $S x x=\sum_{i=1}^{n}\left(X_{i}-\bar{X}\right)^{2}$ and $\bar{X}=\frac{\sum_{i=1}^{n_{1}} X_{i}}{n}, n=$ no. of observations.

The difference $D$ between the two regression lines can then be computed with $\bar{B}$ as the common slope, assuming $\mathrm{B}_{\mathrm{I}}=\mathrm{B}_{\mathrm{II}}=\overline{\mathrm{B}}$

$$
\mathrm{D}=\ln \mathrm{Y}_{\mathrm{I}}-\ln \mathrm{Y}_{\mathrm{II}}=\ln \left(\mathrm{Y}_{\mathrm{I}} / \mathrm{Y}_{\mathrm{II}}\right)-\ln \left(\mathrm{A}_{\mathrm{I}} / \mathrm{A}_{\mathrm{II}}\right)
$$

$$
\text { or } \frac{Y_{I}}{Y_{I I}}=e^{D}=\text { constant. }
$$

$\mathrm{D}$ is the base e logarithm of the ratio of toluene concentration at any time. This is a constant factor which indicates the difference in skin absorption of toluene with and without the barrier cream. Its significance was tested by a $t$ test.

\section{Results}

Table 1 gives the scheme of experiments, with the volunteers' body parameters. Repeated exposure with bare hands showed good reproducibility. Typical curves of elimination are shown (Fig. 1). It should be noted that in some cases the concentration of toluene in alveolar air continues to increase after the end of exposure. This is probably due to physiological properties of the skin. Only three of the 10 subjects showed this phenomenon. The difference in the barrier action of the cream is quite obvious from these charts; a deep application is necessary to obtain the maximum protection. In both cases the skin absorption appears to be much lower when the hands are protected.

All the results relating to the absorption of toluene through the unprotected skin are shown in Figure 2. Individual differences are not very marked, which may be due to a certain uniformity in the absorption rate of toluene in this population.

The results relating to the exposure of protected hands have a larger dispersion of values because the methods of application of the cream are not similar and because the error in the determination of lower toluene concentrations is more important.

Overall results suggest that the cream was effective in preventing toluene absorption. However, a value has to be given to this efficiency in order to compare it with others. Calculations of the regression lines

TABLE 1

Physical Characteristics of 10 Male Subjects studied for Skin Exposure TO LIQUID TOLUENE

\begin{tabular}{l|c|c|c|c|c}
\hline Subject & $\begin{array}{c}\text { Surface area of } \\
\text { one hand }\left(\mathrm{cm}^{2}\right)\end{array}$ & Weight $(\mathrm{kg})$ & Height $(\mathrm{cm})$ & Age (years) & $\begin{array}{c}\text { Mode of } \\
\text { application of } \\
\text { barrier cream }\end{array}$ \\
\hline M.L. & 450 & 62 & 161 & 56 & Deep \\
A.V. & 425 & 60 & 172 & 37 & Deep \\
B.K. & 455 & 79 & 185 & 29 & Deep \\
C.B. & 460 & 83 & 180 & 27 & Deep \\
J.M. & 420 & 70 & 173 & 25 & Deep \\
M.G. & 445 & 65 & 173 & 4 & Superficial \\
C.D. & 465 & 67 & 164 & 43 & Superficial \\
C.P. & 450 & 63 & 175 & 25 & Superficial \\
F.M. & 370 & 65 & 167 & 21 & Superficial \\
M.L. (2) & & & & Superficial \\
\hline
\end{tabular}




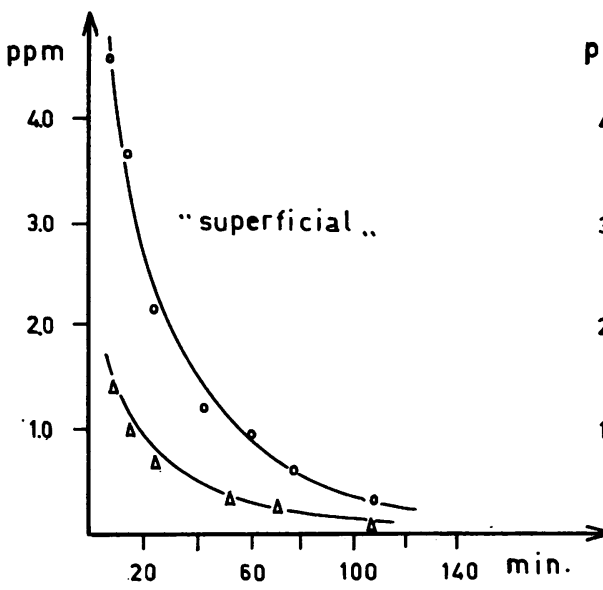

C.P.

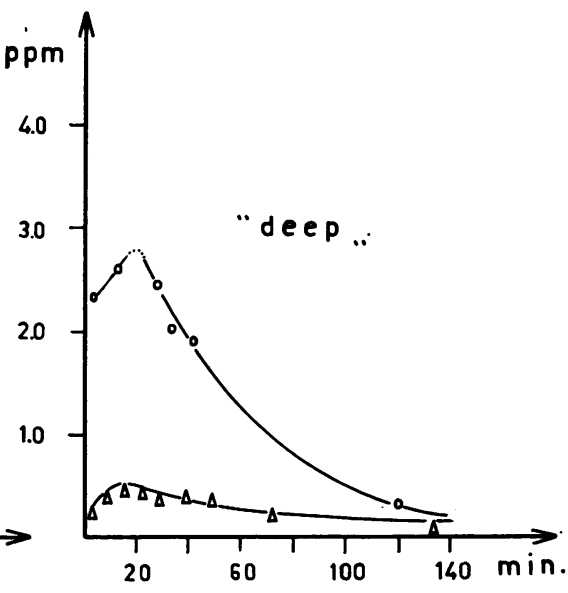

M.L.

FIG. 1. Toluene excretion in alveolar air after skin absorption. Upper curves (-0-0-o-o-) correspond to an exposure of bare hands and lower curves $(-\Delta-\Delta-\triangle-)$ to an exposure of hands protected with a barrier cream. The only difference between the two charts is the mode of application of the barrier cream.

corresponding to all the results with both unprotected and protected skin are shown graphically in Figure 3. It is interesting to note that the slopes of both curves are similar $(P<0.001)$. This tends to show that the rate of exponential elimination is independent of the initial concentrations of the solvent. Numerical results are reported in Table 2.

The distance between the two parallel regression lines was chosen to represent the efficiency of the cream. It is given by the $D$ value. Table 3 gives the mean results obtained for $D$ in each group of experiments and for the corresponding $t$ tests. It is shown that this difference is significant for each group. In the case of superficial application of the cream the dispersion of values between individuals is very large (variance $\mathrm{S}_{0}{ }^{2}=0.33$ ) which causes the difference between the two methods of application to be not significant.

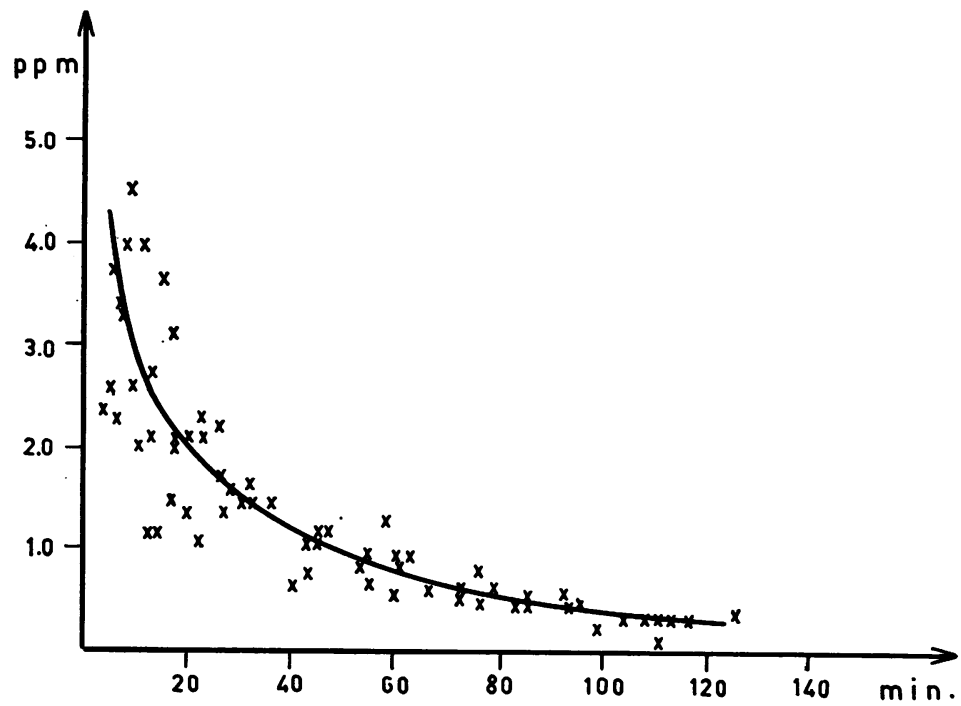

FIG. 2. Toluene excretion in alveolar air after skin absorption. Results of 10 experiments with 10 subjects. The skin of the hands was not protected. 


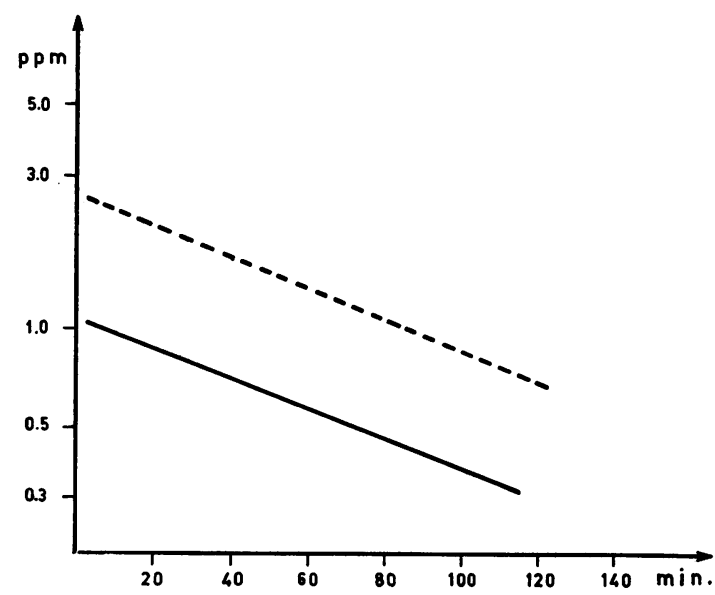

FIG. 3. Regression lines computed from the equation $\mathbf{Y}=\mathrm{A} \mathrm{e}^{\mathrm{Bx}}$. The upper curve (-..-) corresponds to the exposure of bare hands and the lower curve (-) to the exposure of protected hands.

TABLE 2

CONSTANT Factors OF THE Regression Lines $\mathbf{Y}=\mathbf{A e}^{\mathbf{B x}}$ CORRESPONDING TO VARIOUS EXPERIMENTS

\begin{tabular}{l|c|c}
\hline \multicolumn{1}{c|}{ Exposure } & $A($ ppm $)$ & $B$ \\
\hline Without cream & $2.7 \pm 0.9$ & $-0.015 \pm 0.005$ \\
With cream & $1.0 \pm 0.8$ & $-0.014 \pm 0.007$ \\
Total & $0.6 \pm 0.3$ & $-0.011 \pm 0.006$ \\
Deep application & $1.3 \pm 0.9$ & $-0.018 \pm 0.007$ \\
Superficial application & & \\
\hline
\end{tabular}

$\mathrm{X}=$ time after end of exposure (min)

$\mathrm{Y}=$ concentration of toluene in breath $(\mathrm{ppm})$

TABLE 3

Evaluation of the EFficiency OF tHe BarRIER Cream by testing the Mean Difference in SkIn ABSORPTION

\begin{tabular}{l|c|c|c|c|c}
\hline & $\begin{array}{c}\text { No. of } \\
\text { experi- } \\
\text { ments }\end{array}$ & $\begin{array}{c}\mathrm{Y}_{\mathrm{I}}-\mathrm{Y}_{\mathrm{II}} \\
\mathrm{S}^{2} \mathrm{D}\end{array}$ & $\mathrm{t}$ & $\mathrm{P}$ \\
\hline $\begin{array}{c}\text { All experiments } \\
\text { Deep application }\end{array}$ & 10 & 1.16 & 0.19 & 8.41 & $<0.001$ \\
$\begin{array}{l}\text { Superficial appli- } \\
\text { cation }\end{array}$ & 5 & 1.36 & 0.05 & 13.47 & $<0.001$ \\
\hline
\end{tabular}

It should be noted here that the calculated curves do not fit perfectly the measured values in the extremes (short or long time after exposure). The question of finding a perfect mathematical form for the curves is discussed later.

A relative efficiency can be estimated by comparing the surface areas under the elimination curves which are related to the amount of toluene excreted after exposure. These surface areas were calculated as follows:

$$
\begin{gathered}
S=\iint_{x_{1}}^{x_{2}} A^{B x_{1}} d x=\frac{A}{B}\left(e^{B x_{2}}-e^{B x_{1}}\right) \\
x_{1}=20 \text { min } \\
x_{2}=120 \text { min }
\end{gathered}
$$

$\mathrm{S}_{\mathrm{I}}=$ area under elimination curve after exposure with bare hands

$\mathrm{S}_{\mathrm{II}}=$ area under elimination curve after exposure with protected hands

Relative efficiency in percent $=\frac{S_{I}-S_{I I}}{S_{I}} .100$

The results are reported in Table 4.

TABLE 4

Mean Relative Efficiency of the Cream

\begin{tabular}{l|c}
\hline & $\frac{-S_{\text {II }}}{S_{\text {I }}} \times 100$ \\
\hline All experiments & $67 \pm 15 \%$ \\
Deep application & $72 \pm 6 \%$ \\
Superficial application & $61 \pm 20 \%$ \\
\hline
\end{tabular}

A relative efficiency of $0 \%$ would be obtained if there were no difference between the two experiments; $100 \%$ would represent the case where $S_{I I}=0$, i.e., if no toluene could be detected in the breath after exposure of protected skin.

The factor $A$ (concentration of toluene at time zero) was chosen as an index of each individual skin absorption. We attempted to correlate this absorption with other individual parameters, such as age, body weight, height, the quotient weight/height, and the surface area of the hands, to verify whether one of them could be of value in skin absorption. No correlation was found. This fact shows that skin absorption is probably dependent on other factors, such as the type of exposed skin, thickness, vascularity, etc.

It is interesting to compare the elimination of toluene when it is absorbed through the lungs and when it is absorbed through the skin. The mean curve in Fig. 2 is drawn in Fig. 4 in comparison with a curve obtained from Sato and Fujiwara's (1972) results showing the alveolar elimination of toluene after exposure to $100 \mathrm{ppm}$ for 2 hours at rest. 


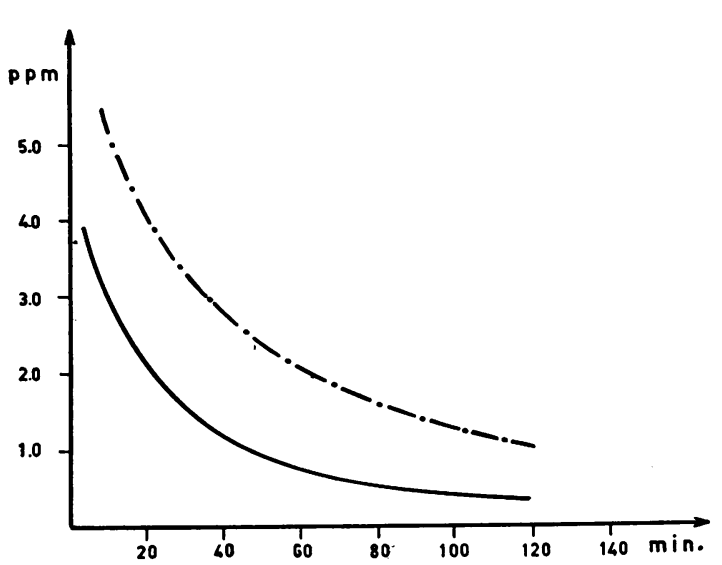

FIG. 4. Comparison between skin absorption (after immersion of both hands in liquid toluene for 10 minutes and lung absorption (-..-.-) after exposure to $100 \mathrm{ppm}$ of toluene for 2 hours (Sato and Fujiwara, 1972).

\section{Discussion}

This study was intentionally limited to the development of a laboratory test which shows the intrinsic efficiency of a barrier cream used against solvents. It may happen that a cream is efficient only for a short time or only under certain working conditions, so that a simple laboratory test would not be sufficient to determine whether a cream is useful or not. But it is possible to use the method presented here in industrial hygiene to study the practical qualities of a cream. It would require only slight modifications such as different breath sampling and different control of exposure to apply the method in the field and to compare skin absorption when the hands are protected or not under various conditions of work. It is obvious that these problems are different in each case and for each type of industry.

The qualitative estimation of skin absorption by breath analysis appears to be very convenient, simple, and rapid compared with other methods such as tracer techniques or urinary analysis of metabolites for instance. This latter method is certainly unsuitable or at least much less appropriate for toluene since it is mainly metabolized to hippuric acid which is a normal constituent of human urine. A difference in the excretion of this acid in urine may be due to other substances than toluene. This makes it difficult to evaluate toluene exposure. For solvents with specific urinary metabolites the method is suitable but it takes a longer time to obtain several urine samples (Dutkiewicz and Tyras, 1967).

According to Stewart and Dodd (1964), 'an estimate of the amount of solvent entering the body through the skin can be made by comparing the post- absorption alveolar breath "decay" curves with "decay" curves obtained following controlled human exposures to known vapour concentrations of the same solvent'. This is true for the solvents that are not transformed in the body (e.g., some halogenated hydrocarbons or anaesthetic agents) but for the others it has been demonstrated (Dutkiewicz and Tyras, 1967) that the metabolic fate of compounds after lung and skin absorption is sometimes tremendously different. This fate is not known for toluene and it is therefore not justifiable to compare the decay curves in order to calculate the absorption rate of liquid toluene through the skin of the hands.

The value of this rate has been found by a direct method to vary between 14 and $23 \mathrm{mg} / \mathrm{cm}^{2} / \mathrm{hr}$ (Dutkiewicz and Tyras, 1968). These data were confirmed in our laboratory. It is possible to calculate from these values the average amount of toluene which entered the body of the subjects when their hands were exposed without any protection. It should be assumed that the absorption rate is not influenced by the surface area exposed.

The calculated amount is $2050 \mathrm{mg}$ to $3370 \mathrm{mg}$ for a mean surface area of both hands of $880 \mathrm{~cm}^{2}$ and an exposure of 10 minutes. In comparison, a calculation of the amount of toluene absorbed through the lungs during an exposure of 2 hours to $100 \mathrm{ppm}$ can be made by using Piotrowski's (1967) data. Assuming a mean retention of $70 \%$ and a mean alveolar breath flow of $260 \mathrm{l} / \mathrm{hr}$, the amount retained would be less than one-tenth of the amount absorbed through the skin. But the postabsorption decay curves show unexpectedly that elimination is much greater after lung exposure (Fig. 4). This could be explained by two hypotheses. The first would be, as already mentioned above, that toluene is metabolized in a different proportion, according to the mode of absorption, as is the case for styrene and ethylbenzene (Dutkiewicz and Tyras, 1967). If this is true, the elimination of toluene in the breath would also be different for each kind of penetration. Absorption through the lungs is rapid because of the large surface area available for solvent penetration and the relative thinness of this tissue barrier as contrasted with that of the skin (Stewart and Dodd, 1964). In one case toluene is absorbed as a vapour diluted in the air and in the other as a pure liquid solvent. There is also a very marked difference between the duration of exposure and probably between the partition coefficients blood-air and blood-lipid. All these facts may be important in the metabolization rate of toluene.

The second hypothesis would be that the assumption of a uniform absorption rate independent of the exposed surface area is not justified. The data employed to calculate the total amount absorbed through the skin (Dutkiewicz and Tyras, 1968) are based on measurements carried out with a surface 
area of $5.55 \mathrm{~cm}^{2}$ lying on the forearm. It may be wrong to extrapolate these values to the whole surface area of the hands. The difference between the skin of the hand and that of the forearm could probably cause different rates of absorption.

The results show that both skin and lung absorptions have to be considered when workers' exposure is assessed, and this cannot be done by breath analysis only. More investigations are necessary, such as careful industrial hygiene surveys with air sampling, study of handling and working conditions, analysis of hippuric acid in urine, and other biological tests completed by a medical examination. Åstrand, Ehrner-Samuel, Kilbom, and Óvrum (1972) demonstrated clearly that breath analysis of toluene cannot be considered as a reliable index of exposure even when only lung absorption occurs. However, it may be very useful as a simple test of absorption under controlled conditions.

In this study the mathematical estimation of the excretion curves was done in a very simple way because a more sophisticated approach was not necessary for our purpose. It was shown for nitrous oxide (Frumin, Salanitre, and Rackow, 1961) and for benzene and toluene (Sato and Fujiwara, 1972) that the excretion curves can be graphically resolved into three exponential components. According to Piotrowski (1971), the mathematical analysis of the excretion rate may be a criterion for determining the number of metabolic compartments of a substance. The number of states in which a substance exists is obtained from the number of phases of the experimental exponential curve. But, whatever relationship each exponential may have to a particular single group of body compartments cannot be reliably evaluated with the data obtained (Frumin et al., 1961). This method of studying metabolic and excretion kinetics of solvents appeared to be very promising, and more research and comprehensive data are still needed.

We wish to thank Professor P. Vuagnat of the University of Geneva for advice and help in the statistical evaluation of the results.

We would like to express our gratitude to $\mathrm{Dr} M$. N. Valloton and Miss E. Brooke of our institute for their assistance.
Our thanks are due to all the volunteers for their kind co-operation.

\section{References}

Åstrand, I., Ehrner-Samuel, H., Kilbom, Å., and O̊vrum, P. (1972). Toluene exposure 1. Concentration in alveolar air and blood at rest and during exercise. Work-Environment-Health, 9, 119-130.

Cooke, M. A. (1966). Skin protection against solvent. Annals of Occupational Hygiene, 9, 215-221.

Dutkiewicz, T. and Tyras, H. (1967). A study of the skin absorption of ethylbenzene in man. British Journal of Industrial Medicine, 24, 330-332.

(1968). Skin absorption of toluene, styrene and xylene by man. British Journal of Industrial Medicine, 25, 243.

Frumin, M. J., Salanitre, E., and Rackow, H. (1961). Excretion of nitrous oxide in anesthetized man. Journal of Applied Physiology, 16, 720-722.

Geigy, J. R. (1972). Tables Scientifiques, pp. 182-183. Ciba-Geigy, Bâle.

Piotrowski, J. (1967). Ilosciowa ocena wchlaniania toluenu u ludzi. Medycyna Pracy, 18, 213-223.

(1971). The Application of Metabolic and Excretion Kinetics to Problems of Industrial Toxicology, pp. 9-28. US Public Health Service, Government Printing Office, Washington DC.

Sato, A. and Fujiwara, Y. (1972). Elimination of inhaled benzene and toluene in man. Japanese Journal of Industrial Health, 14, 224-225.

Schmid, O. (1969). Hautschäden und ihre Verhütung beim Umgang mit Lösemitteln. Berufsdermatosen, 17, 123-135.

Spruit, D., Malten, K. E., Lipmann, E. W. R. M., and The Poo Liang (1970). Horny layer injury by solvents. Berufsdermatosen, 18, 269-280.

Stewart, R. D., and Dodd, H. C. (1964). Absorption of carbon tetrachloride, trichloroethylene, tetrachloroethylene, methylene chloride and 1,1,1-trichloroethane through the human skin. American Industrial Hygiene Association Journal, 25, 439-446.

Wahlberg, J. E. (1971). Absorption-inhibiting effect of barrier creams. Berufsdermatosen, 19, 197-207.

Received for publication 3 September 1973

Accepted for publication 11 December 1973 